\title{
Um método prático de racionamento das aves domésticas *
}

\section{III}

\author{
A. P. TORRES
}

INDICE

1) Relação Cálcio-Fósforo .......... 142

2) Correção da Relação Cálcio-Fósforo ... 144

3) Dedução duma Fórmula Geral . . . . . 145

4) Tabelas $\ldots \ldots \ldots \ldots \ldots \ldots \ldots \ldots \ldots 146$

5) Resumo e Conclusões ........... 149

6) Abstract $\ldots \ldots \ldots \ldots \ldots \ldots \ldots \ldots \ldots 150$

7) Bibliografia $\ldots \ldots \ldots \ldots \ldots \ldots \ldots \ldots 150$

(*) Trabalho da Seção de Avicultura. 


\section{1) RELAÇAO CALCIO-FOSFORO}

Sob o mesmo título dêste trabalho demos à publicidade, nestes mesmas Anais, (1 e 2) um conjunto de regras, formulas e tabelas, que permitiriam a qualquer avicultor o cálculo de formulas cie raçóes devidamente balanceadas, sem as complicaçōes usuais dessas operaçðes, o que the permitiria uma ampla liberdade na utilização das mais variadas forragens disponiveis.

Naquelas publicaçōes, contudo, tratamos apenas do equilibrio protef́co e de regras que mantivessem o coeficiente de empacho adequado, sem fazer referência aos minerais que merecem também consideraçóes, ao lado das vitaminas.

Dêsses minerais é inquestionável a importáncia do cálcio e do fósforo, quer para a saúde, quer para o desenvolvimento, quer para a postura.

Muitos estudos foram feitos neste sentido para estabelecer o quantum de cada um desses elementos deve figurar numa ração.

As conclusões demonstraram que a quantidade de cálcio (CaO) dependia da quantidade de fósforo (P205), havendo uma relação entre esses elementos que tornaria sua utilização mais eficiente.

Para pintos em crescimento, achou-se que a relação favorável estava entre 1.3:1 e 2:1, sendo considerada otima a relação de 1.6:1 (1,6 de cálcio para 1 de fósforo).

Já as aves em pastura têm uma necessidade muito maior de cálcio para a constituiçăo da casca do óvo e essa relaçăo sobe para 2.4:1.

TITUS estabeleceu a seguinte tabela para raçóes de poedeiras: 


\begin{tabular}{c|c|c|c}
\hline \multirow{2}{*}{ Regime só de farelada } & \multicolumn{2}{|c|}{ Regime de farelada e grãos } \\
\hline Cálcio & Fósforo & Cálcio & Fósforo \\
\hline 1.9 & 0.6 & 3.7 & 0.8 \\
2.0 & 0.7 & 3.8 & 0.9 \\
2.1 & 0.8 & 3.9 & 1.0 \\
2.3 & 0.9 & 4.1 & 1.1 \\
2.4 & 1.0 & 4.2 & 1.2 \\
2.5 & 1.1 & 4.3 & 1.3 \\
2.7 & 1.2 & 4.4 & 1.4 \\
2.8 & 1.3 & 4.6 & 1.5 \\
\hline
\end{tabular}

Em regra as rações comuns contém o mínimo do fósforo necessário, que é de $0.7 \%$. Quando êsse número não for atingido, é preciso juntar-se uma pequena dose de farinha de assas. Em geral o cálcio se encontra em quantidade deficiente na ração, sendo necessário juntar-se êsse elemento sob qualquer forma.

Em avicultura, a fonte mais importante de cálcio (CaO) é a farinha de ostra $(38 \%)$ e depois as carbonatos de cálcio de boa qualidade $(39 \%$ de $\mathrm{CaO})$. Excepcionalmente usam-se carbonatos menos ricos em cálcio (de mais de 32\%) e mesmo a cal extinta exposta ao ar durante algum tempo é um recurso para quem não đispõe de calcáreo.

A farinha de ossos, como corretivo das rações em cálcio e fósforo, só é aconselhável quando não se usa na ração a farinha de carne, pois êste produto, em regra, contém dose elevada. de osso moido. Isto constitui, sem dúvida, uma exceção.

Outros minerais de alguma importancia são o cloreto de sódio (sal marinho) e o sulfato de manganês.

A farinha de carne costuma conter uma alta percentagem de sal, porisso, quando ela entra em dose elevada na ração 
(20\%), não se deve juntar mais que $0,5 \%$ de sal na mesma, porém nas rações onde ela figure em proporção até $10 \%$ ou menos, pode-se juntar $1 \%$ de sal com vantagem. O sal impuro é superior ao refinado, por introduzir na ração outros minerais essenciais.

O sulfato de manganês não será necessário se os ingredientes da ração e sobretudo o farelinho de arroz, provém de terras ricas nesse elemento. Durante vários anos nunca observamos, nem em pintos, nem em poedeiras, qualquer sinal de carência dêsse elemento. No entanto, o sulfato de manganês pode ser adicionado à ração sem grandes despesas na proporção de $0,015 \%$, como simples medida de precaução.

\section{2) CORREÇÃO DA RELAÇÃO CALCIO-FÓSFORO}

Para se acertar a relação cálcio-fósforo, é necessário saber-se quanto a ração contem nesses elementos. Afim de omitir cálculos demorados, organizamos uma tabela (I) que dá o número de gs de cálcio (em $\mathrm{CaO}$ ) e fósforo (em P205) para um pêso usual em que o alimento entra numa mistura para formar $100 \mathrm{ks}$ de ração.

Suponhamos que nossa ração consiste de $50 \mathrm{ks}$ de milho, 10 de torta de algodão, 10 de farelinho de arroz, 10 de farelo de trigo, 10 de farinha de carne de $50 \%$ de proteina e 10 de refinazil.

Procurando-se na tabela I, 50 de milho, encontramos os números 5-150, o que quer dizer que $50 \mathrm{ks}$ de milho contêm 5 gs de cálcio e 150 de fósforo. 10 de farelinho de arroz na tabela, figura com 10-184. Ordenando-se todos êstes dados como segue, obteremos o conteúdo total da ração em cálcio e fósforo.

\begin{tabular}{|c|c|c|c|}
\hline 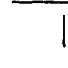 & & $\mathrm{CaO}$ & P205 \\
\hline 50 & ks de milho ....... & 5 & 150 \\
\hline 10 & ks farelo de arroz & 10 & 184 \\
\hline 10 & ks farelo de torta algodão & 22 & 120 \\
\hline 10 & ks farinha de carne $50 \%$. & 1.000 & 500 \\
\hline 10 & $\mathrm{ks}$ farelo de trigo & 11 & 121 \\
\hline 10 & ks farelo de refinazil & 3 & 51 \\
\hline & Conteúdo total & 1.051 & 1.126 \\
\hline
\end{tabular}


Há nessa ração menos cálcio que fósforo. Este acha-se em quantidade mais que suficiente, pois bastariam $0,700 \mathrm{k}$ por $100 \mathrm{ks}$ de ração como mínimo e nós temos, neste caso, $1,126 \mathrm{k}$.

Conforme a ração se destine a pintos em crescimento a relação cálcio-fósforo será $1.6: 1$, enquanto para poedeiras sobe a $2,4: 1$,

Admitamos que a ração se destine a pintos; neste caso, estando satisfeita a necessidade mínima de fósforo, a proporcão de cálcio correspondente, por simples regra de três será:

$$
\text { 1,6:1 : : } \mathrm{x}: 1,126 \quad \mathrm{x}=1,6 \mathrm{x} 1,126=1,802 \mathrm{CaO}
$$

Ora, a ração deveria ter $1,802 \mathrm{k}$ de $\mathrm{CaO}$, porém tem apenas $1,051 \mathrm{k}$, faltando portanto $0,751 \mathrm{k}$ de $\mathrm{CaO}$.

Se êsse cálcio tiver que ser fornecido por farinha de ostra (com 38\% de CaO) a quantidade de farinha de ostra a ser adicionada será:

Se um quilo de farinha de ostra contêm $0,380 \mathrm{k}$ CaO, quantos quilos de farinha de ostra darão 0,751 k CaO ou

$$
\text { 1: } 0,380:: x: 0.751 \quad x=\frac{0.751}{0,380}=1,976 \mathrm{ks} \sim 2 \mathrm{ks}
$$

\section{3) DEDUÇAO DUMA FORMULA GERAL}

As operações se resumem em multiplicar o conteúdo em fósforo $(1,126)$ pela relação desejada $(1,6)$ deduzindo dêsse total o cálcio já existente (1.051) e dividindo-se o resultado pelo teor em cálcio do calcáreo usado $(0,380)$.

Uma fórmula geral para determinar a quantidade de uma fonte de calcio qualquer (exceto fosfatos) para fornecer 0 CaO necessário ao equilíbrio de uma ração seria:

$$
\mathrm{Qca}=\frac{(\operatorname{Rca} \times \mathrm{Tp})-\mathrm{Tca}}{\% \mathrm{ca}}
$$

na qual Qca = quantidade de farinha de ostra ou outro calcáreo a adicionar.

Rca = proporção de cálcio desejada para 1 de fósforo.

$T p=$ total de fósforo já existente na ração.

Tca = total de cálcio já existente na ração.

$\%$ ca $=$ teor em cálcio da farinha de astra ou cálcareo a usar. 
Aplicando esta fórmula ao nosso exemplo, teríamos:

$$
\mathrm{Qca}=\frac{(1,6 \times 1,126)-1,051}{0,380}=1,976 \sim 2 \mathrm{ks}
$$

Se a ração destinasse a poedeiras, a relação de cálcio desejada seria 2,4, então a quantidade de farinha de ostra seria:

$$
\mathrm{Qca}=\frac{(2,4 \times 1,126)-1,051}{0,380}=4.345: \sim 4,500 \mathrm{ks}
$$

Fórmulas, como estas, têm a vantagem de dispensar o raciocínio e constituem uma vantagem prática, mas não eliminam cálculos.

Achamos que seria muito mais interessante a apresentação de tabelas que dessem diretamente a quantidade de farinha de ostra a ser empregada para corrigir uma determinada deficiência.

\section{4) TABELAS}

A organização duma tabela que desse a quantidade de farinha de ostra para completar o cálcio necessário para equilibrar uma ração foi operação simples. Considerando que para fornecer $0,100 \mathrm{k}$ de cálcio são necessários $0,263 \mathrm{k}$ de farinha de ostra, determinamos o número de ks de ostra suficientes para fornecer o cálcio necessário ao balanço. Vejam-se tabelas II e III.

Acreditamos que a tabela pudesse ser muito reduzida, mas só deixamos extensa para satisfazer a hipóteses pouco frequentes. Também achamos absurdo o emprêgo de mais de $6 \%$ de concha de astra numa ração, porisso paramos nesse limite.

O número de quilos de farinha de ostra é dado com aproximação, mas perfeitamente satisfatório. Se tomarmos o nosso exemplo citado anteriormente, para poedeiras, o conteúdo da ração em cálcio e fósforo era respectivamente de $1,051 \mathrm{k}$ e $1,126 \mathrm{k}$; procurando na tabela II - para poedeiras - $1,0 \mathrm{k}$ de cálcio e 1,1 de fósforo, achamos 4,0, ou sejam, $4 \mathrm{ks}$ de farinha de ostra, quando a determinação exata deu 4,345

Essa pequena diferença não tem nenhuma importância prática, e os dados das tabelas podem ser usados com segurança. 
TABELA I

Conteúdo em cálcio e fósforo ( $\mathrm{CaO}-\mathrm{P2O5}$ ) nas quantidades usuais dos alimentos Ks. de alimento contém grs. de cálcio-fósforo

\begin{tabular}{|c|c|c|c|c|c|c|c|c|c|}
\hline Alimentos em pequena $\%$ & 1 & 2 & 3 & 4 & 5 & 7 & 10 & 12 & 15 \\
\hline $\begin{array}{l}\text { Alfafa, farinha de folhas } \ldots \ldots \ldots \\
\text { Algodão, far. de torta } \ldots \ldots \ldots \ldots \\
\text { Amendoim, farelo de } \ldots \ldots \ldots \ldots \\
\text { Arroz, farelinho de } \ldots \ldots \ldots \ldots \ldots \\
\text { Babaçú, farelo de } \ldots \ldots \ldots \ldots \ldots\end{array}$ & $\begin{array}{l}19-2 \\
2-12 \\
2-6 \\
1-18 \\
3-7\end{array}$ & $\begin{array}{r}38-4 \\
4-24 \\
4-11 \\
2-37 \\
6-13\end{array}$ & $\begin{array}{r}57-7 \\
6-36 \\
6-17 \\
3-55 \\
9-20\end{array}$ & $\begin{array}{r}76-9 \\
8-48 \\
8-23 \\
4-74 \\
12-27\end{array}$ & $\begin{array}{r}95-11 \\
10-60 \\
9-29 \\
5-92 \\
15-34\end{array}$ & $\begin{array}{c}33-15 \\
14-84 \\
13-40 \\
7-129 \\
21-47\end{array}$ & $\begin{array}{l}190-22 \\
22-120 \\
18-57 \\
10-184 \\
30-67\end{array}$ & $\begin{array}{l}26-144 \\
22-68 \\
12-221 \\
36-80\end{array}$ & $\begin{array}{l}32-180 \\
27-86 \\
15-276 \\
45-100\end{array}$ \\
\hline 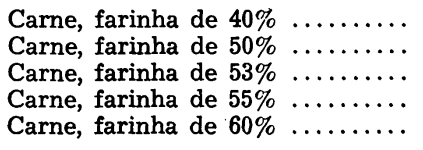 & $\begin{array}{r}320-160 \\
100-50 \\
138-69 \\
80-40 \\
72-35\end{array}$ & $\begin{array}{l}640-320 \\
200-100 \\
276-138 \\
160-80 \\
144-70\end{array}$ & $\begin{array}{l}960-480 \\
300-150 \\
414-207 \\
240-120 \\
216-105\end{array}$ & $\begin{array}{r}1280-640 \\
400-200 \\
552-276 \\
320-160 \\
286-140\end{array}$ & $\begin{array}{r}1600-800 \\
500-250 \\
690-345 \\
400-200 \\
358-177\end{array}$ & $\begin{array}{r}1440-720 \\
700-350 \\
966-483 \\
560-280 \\
512-247\end{array}$ & $\begin{array}{l}3200-1600 \\
1000-500 \\
1380-690 \\
800-400 \\
716-353\end{array}$ & $\begin{array}{c}3840-1920 \\
1200-600 \\
1656-828 \\
960-480 \\
870-423\end{array}$ & $\begin{array}{l}4800-2400 \\
1500-750 \\
2070-1035 \\
1200-600 \\
1074-530\end{array}$ \\
\hline $\begin{array}{l}\text { Côco, farelo de } \ldots \ldots \ldots \ldots \ldots \ldots \\
\text { Fermento } \ldots \ldots \ldots \ldots \ldots \ldots \ldots \\
\text { Gergelim, farelo de } \ldots \ldots \ldots \ldots \ldots \\
\text { Girassol } \ldots \ldots \ldots \ldots \ldots \ldots \ldots \\
\text { Girassol, farelo de torta } \ldots \ldots \ldots\end{array}$ & $\begin{array}{c}2-6 \\
3-50 \\
20-16 \\
1-6 \\
6-22\end{array}$ & $\begin{array}{c}4-12 \\
6-100 \\
40-32 \\
2-11 \\
11-43\end{array}$ & $\begin{array}{r}6-18 \\
9-150 \\
60-48 \\
3-17 \\
17-65\end{array}$ & $\begin{array}{l}8-25 \\
12-200 \\
80-64 \\
4-22 \\
22-86\end{array}$ & $\begin{array}{c}10-31 \\
15-250 \\
100-80 \\
5-28 \\
28-108\end{array}$ & $\begin{array}{c}15-43 \\
21-350 \\
140-112 \\
8-39 \\
39-151\end{array}$ & $\begin{array}{c}21-62 \\
30-500 \\
202-161 \\
11-55 \\
55-215\end{array}$ & $\frac{\frac{25-74}{242-193}}{66-258}$ & $\frac{\frac{31-93}{303-241}}{83-323}$ \\
\hline $\begin{array}{l}\text { Guandú } \ldots \ldots \ldots \ldots \ldots \ldots \ldots \ldots \\
\text { Leite desnatado sêco } \ldots \ldots \ldots \ldots \ldots \\
\text { Ossos, farinha de } \ldots \ldots \ldots \ldots \ldots \ldots \\
\text { Ostras, farinha de } \ldots \ldots \ldots \ldots \ldots \ldots \\
\text { Soja, grãos } \ldots \ldots \ldots \ldots \ldots \ldots \\
\text { Soja, farelo de torta } \ldots \ldots \ldots \ldots \ldots\end{array}$ & $\begin{array}{l}13-10 \\
330-150 \\
380-0 \\
2-5 \\
3-7\end{array}$ & $\begin{array}{l}25-19 \\
660-300 \\
760-0 \\
4-11 \\
6-13\end{array}$ & $\begin{array}{c}39-29 \\
990-450 \\
1140-0 \\
6-16 \\
8-20\end{array}$ & $\begin{array}{c}51-38 \\
1320-600 \\
1520-0 \\
8-21 \\
11-26\end{array}$ & $\begin{array}{c}64-48 \\
1650-750 \\
1900-0 \\
10-27 \\
14-33\end{array}$ & $\frac{77-67}{\overline{14-37}}$ & $\frac{127-96}{\overline{20-53}}$ & $\frac{152-115}{\square}$ & $\frac{190-144}{\square}$ \\
\hline Alimentos em grande $\%$ & 7 & 10 & 12 & 15 & 20 & 30 & 40 & 50 & 60 \\
\hline 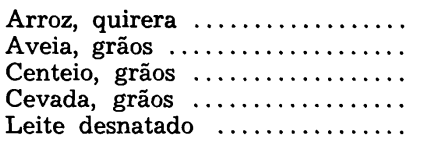 & $\begin{array}{l}1-16 \\
7-25 \\
3-25 \\
3-25 \\
10-8\end{array}$ & $\begin{array}{r}1-9 \\
10-36 \\
5-36 \\
5-36 \\
14-12\end{array}$ & $\begin{array}{r}1-11 \\
12-43 \\
6-43 \\
6-43 \\
17-14\end{array}$ & $\begin{array}{r}2-14 \\
15-54 \\
7-54 \\
7-54 \\
21-18\end{array}$ & $\begin{array}{r}2-18 \\
20-72 \\
10-72 \\
10-72 \\
28-24\end{array}$ & $\begin{array}{l}3-27 \\
30-108 \\
15-108 \\
15-108 \\
42-36\end{array}$ & $\bar{\square} \bar{\square}$ & $\overline{\bar{L}}$ & $\bar{\square}$ \\
\hline $\begin{array}{l}\text { Mandioca, raspas } \ldots \ldots \ldots \ldots \ldots \ldots \\
\text { Mandioca, farelo } \ldots \ldots \ldots \ldots \ldots \ldots \\
\text { Melaço } \ldots \ldots \ldots \ldots \ldots \\
\text { Milho, grâo ou fubá } \ldots \ldots \ldots \ldots \ldots \\
\text { Milho moido com sabugo } \ldots \ldots \ldots\end{array}$ & $\begin{array}{c}18-20 \\
39-4 \\
1-21 \\
7-61\end{array}$ & $\begin{array}{l}25-29 \\
56-6 \\
1-30 \\
10-85\end{array}$ & $\begin{array}{c}30-25 \\
67-7 \\
1-36 \\
12-102\end{array}$ & $\begin{array}{c}38-44 \\
84-9 \\
2-45 \\
15-128\end{array}$ & $\begin{array}{c}50-58 \\
20-60 \\
20-170\end{array}$ & $\begin{array}{l}75-87 \\
3-90 \\
\end{array}$ & $\frac{100-116}{4-120}$ & $\overline{5-150}$ & $\overline{6-180}$ \\
\hline 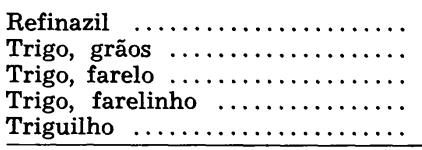 & $\begin{array}{r}2-36 \\
6-29 \\
8-85 \\
19-39 \\
6-50\end{array}$ & $\begin{array}{c}3-51 \\
8-93 \\
11-121 \\
28-93 \\
9-70\end{array}$ & $\begin{array}{l}4-61 \\
10-112 \\
13-145 \\
34-112 \\
11-84\end{array}$ & $\begin{array}{c}5-77 \\
12-139 \\
17-182 \\
42-139 \\
14-105\end{array}$ & $\begin{array}{r}6-102 \\
16-186 \\
22-242 \\
56-186 \\
18-140\end{array}$ & $\begin{array}{r}9-153 \\
24-279 \\
33-363 \\
84-279 \\
27-210\end{array}$ & $\begin{array}{r}12-204 \\
44-482 \\
112-372 \\
\end{array}$ & & {[} \\
\hline
\end{tabular}




\section{TABELA II}

\section{Rações para poedeiras}

(No cruzamento da coluna com a linha, a quantidade de farinha de ostra a adicionar

Conteúdo em P205 na ração

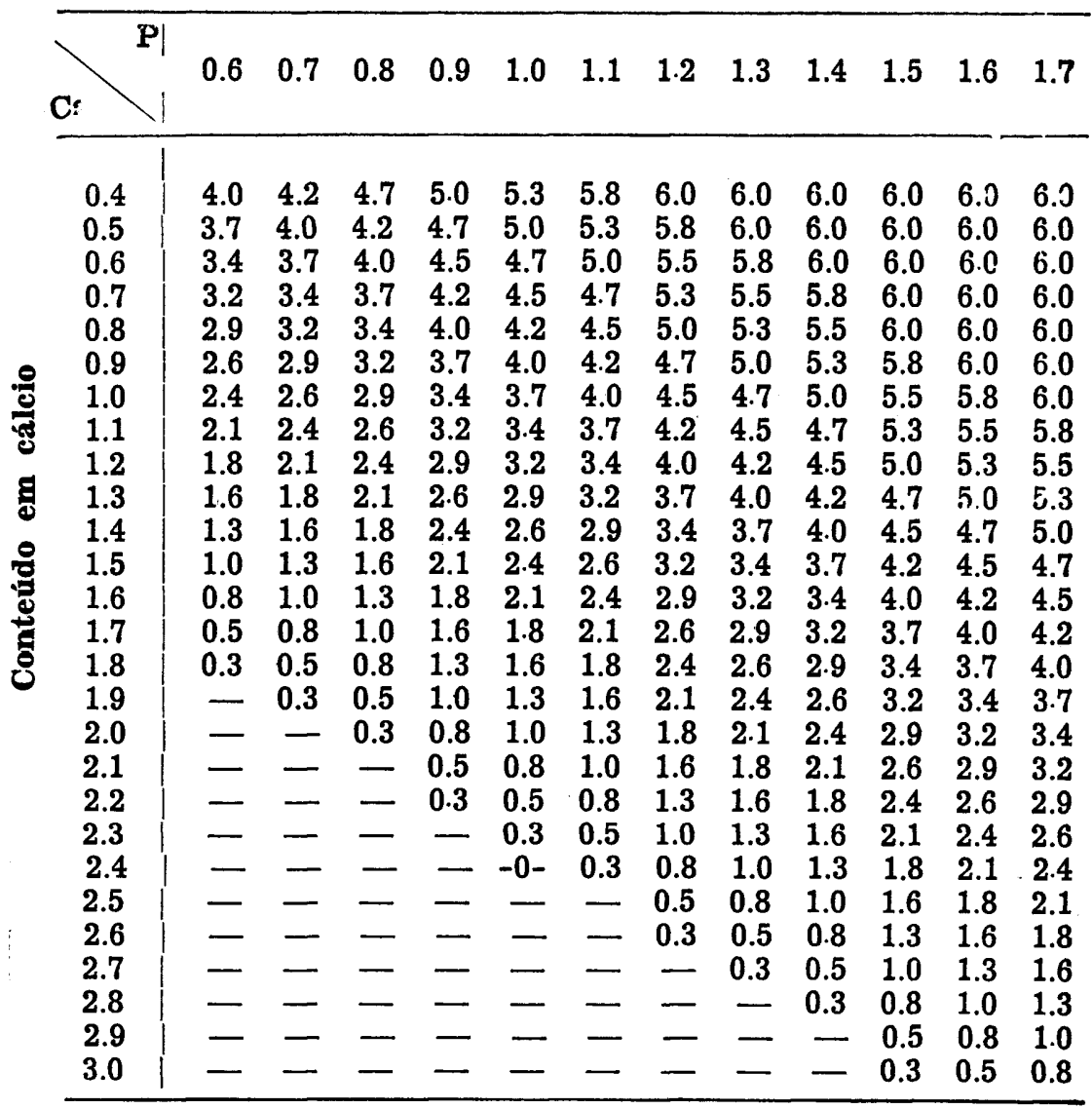


Anais da E. S. A. "Luiz de Queiroz"

\section{TABELA III}

Rações para crescimento

(No cruzamento das linhas com as colunas, a quantidade de farinha de ostra a adicionar)

\section{Conteúdo em P205 na ração}

\begin{tabular}{|c|c|c|c|c|c|c|c|c|c|c|c|c|}
\hline 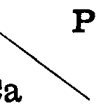 & 0.6 & 0.7 & 0.8 & 0.9 & 1.0 & 1.1 & 1.2 & 1.3 & 1.4 & 1.5 & 1.6 & 1.7 \\
\hline 0.4 & 2.1 & 2.4 & 2.6 & $2.9^{-}$ & 3.2 & 3.4 & 3.7 & 4.0 & 4,2 & 4.5 & 4.7 & 5.0 \\
\hline 0.5 & 1.8 & 2.1 & 2.4 & 2.6 & 2.9 & 3.2 & 3.4 & 3.7 & 4.0 & 4.2 & 4.5 & 4.7 \\
\hline 0.6 & 1.6 & 1.8 & 2.1 & 2.4 & 2.6 & 2.9 & 3.2 & 3.4 & 3.7 & 4.0 & 4.2 & 4.5 \\
\hline 0.7 & $1: 3$ & 1.6 & 1.8 & 2.1 & 2.4 & 2.6 & 2.9 & 3.2 & 3.4 & 3.7 & 4.0 & 4.2 \\
\hline 0.8 & 1.0 & 1.3 & 1.6 & 1.8 & 2.1 & 2.4 & 2.6 & 2.9 & 3.2 & 3.4 & 3.7 & 4.0 \\
\hline 0.9 & 0.8 & 1.0 & 1.3 & 1.6 & 1.8 & 2.1 & 2.4 & 2.6 & 2.9 & 3.2 & 3.4 & 3.7 \\
\hline 1.0 & 0.5 & 0.8 & 1.0 & 1.3 & 1.6 & 1.8 & 2.1 & 2.4 & 2.6 & 2.9 & 3.2 & 3.4 \\
\hline 1.1 & 0.3 & 0.5 & 0.8 & 1.0 & 1.3 & 1.6 & 1.8 & 2.1 & 2.4 & 2.5 & 29 & 3.2 \\
\hline 1.2 & - & 0.3 & 0.5 & 0.8 & 1.0 & 1.3 & 1.6 & 1.8 & 2.1 & 2.4 & 2.6 & 2.9 \\
\hline 1.3 & - & - & 0.3 & 0.5 & 0.8 & 1.0 & 1.3 & 1.6 & 1.8 & 2.1 & 2.4 & 2.6 \\
\hline 1.4 & - & - & - & 0.3 & 0.5 & 0.8 & 1.0 & 1.3 & 1.6 & 1.8 & 2.1 & 2.4 \\
\hline 1.5 & - & - & - & - & 0.3 & 0.5 & 0.8 & 1.0 & 1.3 & 1.6 & 1.8 & 2.1 \\
\hline 1.6 & - & - & - & - & $-0-$ & 0.3 & 0.5 & 0.8 & 1.0 & 1.3 & 1.6 & 1.8 \\
\hline 1.7 & - & - & - & - & - & - & 0.3 & 0.5 & 0.8 & 1.0 & 1.3 & 1.6 \\
\hline 1.8 & - & - & - & - & - & - & - & 0.3 & 0.5 & 0.8 & 1.0 & 1.3 \\
\hline 1.9 & - & - & - & - & - & - & - & - & 0.3 & 0.5 & 0.8 & 1.0 \\
\hline 2.0 & - & - & $=$ & - & - & - & - & $=$ & 二 & 0.3 & 0.5 & 0.8 \\
\hline 2.1 & - & - & - & - & - & - & - & - & - & - & 0.3 & 0.5 \\
\hline
\end{tabular}




\section{5) RESUMO E CONCLUSÕES}

O A., que já apresentou um método rápido para cálculo de ração, no qual faltava o balanço Cálcio-Fósforo, apresenta neste trabalho duas maneiras de conseguir isso com rapidez e facilidade:

(a) por meio de uma fórmula

$$
\mathrm{Qca}=\frac{(\text { Rca } \times \mathrm{Tp})-\mathrm{Tca}}{\% \mathrm{ca}} \text { na qual, Qca é a quantidade }
$$

de farinha de ostra ou outro calcáreo a adicionar, a ser determinada; Rca, proporção de cálcio (CaO) desejada para 1 de fósforo (P2O5); Tp, total de P2O5 já existente na ração; Tca, total de cáleio fá existente na ração e \% ca teor em cálcio do calcáreo que se vaí usar para corrigir a relação: $\mathrm{CaO} / \mathrm{P2O5}$.

b) por meio de tabelas adrede preparadas, especiais para aplicação de farinha de os'ra. Procurando-se nas linhas o teor mais aproximado do fósforo existente na ração e nas colunas a de cálcio já existente na ração; na sua interseção se encontra a quantidade em ks de ostra que deve ser adicionada a $100 \mathrm{ks}$ de ração, para cobrir o déficit de cálcio.

O A. como elemento auxiliar apresenta também uma tabela dos alimentos utilizados na região com o conteúdo em Ca e $P$, para as percentagens mais usuais em que entram numa ração, dispensando desta maneira os cálculos habituais.

A principal característica do método é a rapidez extraordinária como que se opera, evitando os naturais erros de cálculo possiveis para os práticos pouco afeitas a êsses trabalhos.

Finalmente, nossa experiência em cálculos frequentes de rações, em nossa condições ordinárias, chegamos à conclusão, que em média deve-se, a grosso modo, juntar às rações $2 \mathrm{ks}$ de farinha de ostra para as aves em crescimento e $4 \mathrm{ks}$ para as poedeiras, sem afetar muito a conveniente relação cálciofósforo. Isto sempre que não se possa fazer cálculos ou usar desta tabela. 


\section{6) ABSTRACT}

A short method of ration formula determination was already presented by the author $(1,2)$ in which the ratio calcium-phasphorus was lacking. The present paper deals with two ways in finding the relation quickly:

a) by means of a formula

$$
\mathrm{Qca}=\frac{(\mathrm{Rca} \times \mathrm{Tp})-\mathrm{Tca}}{\% \mathrm{ca}}
$$

where QCa = the quantity of oyster shell or other limestone to be determined; $\mathrm{Rca}=$ the calcium ratio $(\mathrm{CaO})$ desired for 1 of the phosphorus; $\mathrm{Tp}=$ the total of P2O5 found in the ration; $\mathrm{TCa}=$ the total calcium found in the ration and $\% \mathrm{ca}=$ the amount of the calcium present in the source of calcium to be used in correcting the relation CaO/P2O5.

b) by means of proper calculated tables for oyster shell. The horizontal lines show the approximate amount of phosphorus present in the ration and the coluns the same for calcium. In the intersection of the two lines we find in kgs the amount of oyster shell necessary to be added to $100 \mathrm{kgs}$ of ration in order to recover the calcium deficit.

A table containing the amount in grs. of $\mathrm{Ca}$ and $\mathrm{P}$ of a given percentage of the main feeds used in the country is presented.

The main feature of the method is the quickly determination of the subject without possible errors of calculation.

\section{7) BIBLIOGRAFIA}

1) TORRES, A. P., 1946. Um método prático de racionamento das aves domésticas. Anais da Escola Superior de Agricultura "Luiz de Queiroz", 3:313-322. Piracicaba. 
2) TORRES, A. P., 1946. Um método prático de racionamento das aves domésticas. Nota Suplementar. Anais da Escola Superior de Agricultura "Luiz de Queiroz", 3:323-328. Piracicaba.

3) TITUS, H. W., 1939. Food and Life. Yearbook of Agric., U. S. Dep. Agr., Washington.

4), HOLST, W. F. e NEWLON W E., 1935. Poultry Feeding, Rev. H-J. Almquist e I. H. Jukes. Bol. 417 - Univ. California, Berkeley.

5) WILGUS JR., H. S., 1931. The quantitative requirement of the growing chick for calcium and phasphorus, Poultry Sc. $\mathrm{X}: 107-117$.

6) JULL, M. A., 1938. Poultry Husbandry. 2." Ed. 6." Imp. N. York, Londres. 
$\cdots \cdots \cdots$ 